\title{
Combination of new multifunctional molecules for erythematotelangiectatic rosacea disorder
}

This article was published in the following Dove Press journal:

Clinical, Cosmetic and Investigational Dermatology

I October 2015

Number of times this article has been viewed

\author{
Hanane Chajra' \\ Mahdi Nadim² \\ Daniel Auriol ${ }^{3}$ \\ Kuno Schweikert ${ }^{\prime}$ \\ Fabrice Lefevre' \\ 'Induchem AG, Volkestwil, Switzerland; \\ ${ }^{2} \mathrm{CBM}-\mathrm{CNRS}$, Orléans Cedex, \\ ${ }^{3}$ Libragen SA, Toulouse, France
}

Correspondence: Hanane Chajra Induchem AG, Industriestrasse 8a, $\mathrm{CH}-8604$ Volkestwil, Switzerland Email hchajra@induchem.com
Background: Rosacea, a common chronic skin disorder, is currently managed by patient education, pharmacological drugs, medical devices (laser and light therapies), and use of proper skin cares. Unfortunately, none of these actual treatments used alone or in combination is curative, and so we proposed a dermocosmetic active ingredient to mitigate some aspects of the rosacea and particularly for erythematotelangiectatic rosacea.

Methods: Dermocosmetic active ingredient is composed of three glucosylated derivatives of natural plants hydroxybenzoic acid and hydroxycinnamic acids (rosmarinic acid, gallic acid, and caffeic acid). Anti-inflammatory, anti-angiogenesis, and anti-degranulation studies were done on cellular models (keratinocytes, mast cells, and endothelial cells). Efficiency of the active ingredient in comparison to placebo was assessed clinically on human volunteers having erythematotelangiectatic rosacea. The active and placebo were applied topically twice a day for 28 days. Biometrical analyses were done using a siascope tool.

Results: We found that the active ingredient decreases inflammation (inhibition of interleukin- 8 and tumor necrosis factor release), decreases degranulation of mast cells (inhibition of histamine release), and controls angiogenesis mechanism (inhibition of the production of vascular endothelial growth factor and neovessel formation) on cellular models. Study on human volunteers confirmed macroscopically the efficiency of this active ingredient, as we observed no neovessel formation and less visible vessels.

Conclusion: Although rosacea is a skin condition disorder that is difficult to heal, the studies have shown that this active ingredient could be a dermocosmetic support, especially for erythematotelangiectatic rosacea armamentarium. The active ingredient was topically applied on the face for 28 days and improved erythematotelangiectatic rosacea symptoms either by decreasing them (vessels are less visible) or by limiting their development (any neovessels). The active ingredient decreases inflammation (inhibition of interleukin- 8 and tumor necrosis factor release), decreases degranulation of mast cells (inhibition of histamine release), and limits the angiogenesis process (inhibition of vascular endothelial growth factor production and neovessel formation).

Keywords: rosacea, erythema, gallic acid, rosmarinic acid, caffeic acid, glucosylation

\section{Introduction}

Rosacea is a common chronic skin disorder mainly affecting areas of skin having a high density of sebaceous glands, such as cheeks, nose, chin, and forehead. ${ }^{1}$ Pathogenesis of rosacea was described as an inflammatory vasculopathy, ${ }^{2}$ involving mast cells and vascular endothelial growth factor (VEGF). Mast cells trigger more inflammation, promoting vasodilatation and angiogenesis. ${ }^{3}$ VEGF induces the proliferation of vascular endothelial cells and increases vessels permeability and cellular infiltration. ${ }^{4}$ Many 
studies demonstrated that Rosacea has three stages and an ocular one classified in the following four subtypes: subtype 1 with flushing or transient erythema (erythematotelangiectatic rosacea); subtype 2 with persistent erythema and transient papules and pustules (papulopustular acne rosacea); subtype 3 with thickening of the skin (phymatous or rinofima rosacea); and subtype 4 with eye dryness, irritation, and conjunctivitis (ocular rosacea). There is no clear continuum from one subtype to another, ie, one's condition could worsen from an erythematous rosacea to a rinofima without passing by the subtype 2 or even can start up with a papulopustular stage.

Based on these known physiopathological origins of rosacea, we developed an active ingredient acting on degranulation, inflammation, and angiogenesis processes. Its efficacy has been tested in vitro on specialized cells, such as normal keratinocytes, normal mast cells, and skin endothelial cells. It was finally assessed on human volunteers having erythematotelangiectatic rosacea (subtype 1).

\section{Materials and methods Material tested}

The active ingredient is water soluble and is obtained by enzymatic modification of natural plants' hydroxybenzoic acid and hydroxycinnamic acids, such as rosmarinic acid, gallic acid, and caffeic acid, using a patented process. ${ }^{5}$ These three molecules were glucosylated by biocatalytic reaction. In contrast to the nonglucosylated forms, the glucosylated derivatives are stable, water soluble, and easy to formulate.

The active ingredient is the combination of the stabilized glucosylated derivatives of these three molecules.

The active ingredient is identified under the denomination RGC for rosmarinic acid glucoside ( $R$ ), gallic acid glucoside $(\mathrm{G})$, and caffeic acid glucoside (C) (International Nomenclature of Cosmetic Ingredients: water, glycerin, rosmarinyl glucoside, caffeyl glucoside, and gallyl glucoside; trade name Endothelyo ${ }^{\circledR}$ ).

RGC mother solution is composed of $25.2 \mathrm{mM}$ rosmarinic acid glucoside, $19.6 \mathrm{mM}$ gallic acid glucoside, and $28.6 \mathrm{mM}$ caffeic acid glucoside. RGC was tested in vitro on cellular models diluted at $1 \%, 1.5 \%$, and $2.5 \%$ in culture media and in vivo on human volunteers formulated in a serum at $2 \%$. The full serum composition is given in the "In vivo study" section.

The study was performed in accordance with the Declaration of Helsinki after the patients had given written informed consent.

\section{Cytotoxicity assays}

The potential cytotoxicity of RGC was assessed by various methods depending on the cell types studied: trypan blue ${ }^{6}$ (mast cells), 2,3-Bis-(2-Methoxy-4-Nitro-5Sulfophenyl)-2H-Tetrazolium-5-Carboxanilide (XTT) $)^{7,8}$ (keratinocytes), and alamar blue ${ }^{9}$ (endothelial cells).

\section{Cytokines release assays (anti- inflammatory studies): measure of tumor necrosis factor alpha and interleukin-8}

\section{Products and references}

Interleukin-1 beta (IL-1 beta; recombinant human IL-1 beta, 201-LB; R\&D Systems, Inc., Minneapolis, MN, USA) was used to stimulate IL-8 release, and phorbol myristate acetate (PMA, P-8139; Sigma-Aldrich Co, St Louis, MO, USA) was used to stimulate tumor necrosis factor alpha (TNF alpha) release from keratinocytes. Dexamethasone (D-4902; SigmaAldrich Co) was used as positive control for IL-8 and TNF alpha release inhibitions.

\section{Cell treatments}

Human normal keratinocytes (isolated from biopsy obtained from surgical operation) were seeded in 96-well microplate at the density of 20,000 cells/well (culture medium keratinocyte serum free medium [KSFM]; Thermo Fisher Scientific, Waltham, MA, USA). Plate was placed in an incubator maintained at $37^{\circ} \mathrm{C}$ and $5 \% \mathrm{CO}_{2}$ until cells are confluent at $80 \%$. Cells were pretreated at different concentrations of $\mathrm{RGC}(1 \%, 1.5 \%$, and $2.5 \%)$ in culture medium for 24 hours and then stimulated with IL-1 beta ( $1 \mathrm{ng} / \mathrm{mL}$ for IL-8 release) or PMA at $500 \mathrm{ng} / \mathrm{mL}$ (for TNF alpha release) during 24 hours. Each product concentration was evaluated in triplicate. Cells were kept without treatment for control condition. At the end of the experiment, cell supernatants were frozen at $-20^{\circ} \mathrm{C}$ for subsequent cytokines assay.

\section{Cytokines assays}

Cytokines were measured as described in the Quantikine human IL-8 (D8000C; R\&D Systems) and in the Quantikine human TNF alpha (DTA00C; R\&D Systems). The spectrophotometric absorbance was measured using a scanning multiwell spectrophotometer (Safire; Tecan, Mannedorf, Switzerland). The wavelength to measure absorbance of the substrate was $450 \mathrm{~nm}$. A best fit curve was generated for the standards in order to extrapolate relative sample values. The amounts of IL-8 and TNF alpha were expressed in pg/ $\mathrm{mL}$. A protein assay was done for every well of the plate. The percentages of inhibition under stimulated conditions induced by the treatment were calculated for each concentration of RGC. 


\section{Protein quantification}

The bicinchoninic acid assay, which is similar to the Lowry procedure, was selected for measuring the concentrations of synthesized proteins. Determination of protein quantity was performed in each well (cell lysate) for which supernatant was quantified for cytokines. Values were expressed in $\mu \mathrm{g}$ protein. Therefore, final results for IL-8 or TNF alpha were expressed in $\mathrm{pg} / \mathrm{mL} / \mu \mathrm{g}$ protein. Values from three wells treated with the same condition were averaged. This average was compared with control average (cells kept without treatment).

\section{Histamine release assay on mast cells}

Product and references

Substance P is a neuropeptide purchased from NeoMPS (SC000; Strasbourg, France). Substance P was used at $50 \mu \mathrm{M}$.

RGC was prepared from a sterile stock solution and tested at $0.25 \%, 1.5 \%$, and $2.5 \%$ (v/v dilutions in medium culture). A $10 \mathrm{mM}$ calcium solution was used as inhibitory control.

\section{Treatment and stimulation of mast cells}

Mammalian cells were purchased from Charles Rivers (France). Mast cells $\left(1 \times 10^{5}\right.$ cells $/ \mathrm{mL}$ or $5 \times 10^{4}$ cells/tube) were incubated at $37^{\circ} \mathrm{C}$ in the presence or absence of the products for 30 minutes and then stimulated by substance $\mathrm{P}$ for 15 minutes. The stimulations were stopped by centrifugation at $4^{\circ} \mathrm{C}$.

\section{Histamine release}

The reaction was stopped by centrifugation $(1,800 \mathrm{rpm}, 10 \mathrm{~min}-$ utes at $\left.4^{\circ} \mathrm{C}\right)$. Supernatants and cell pellets are suspended in perchloric acid $0.4 \mathrm{~N}$. The measurement was performed with an enzyme-linked immunosorbent assay kit Histamine (RE59221; IBL Hamburg). Each condition (product and controls) was done in triplicate. The percentage of release inhibition was then calculated.

\section{Angiogenesis studies using keratinocytes and endothelial cells}

Product and references for VEGF quantification assay RGC under liquid solution was tested at $1 \%, 1.5 \%$, and $2.5 \%$. Recombinant human IL-1 beta at $10 \mathrm{ng} / \mathrm{mL}$ (201-LB) was used for VEGF cell stimulation. Dexamethasone (D-4902) at $10 \mu \mathrm{M}$ was used as inhibitor of VEGF release.

\section{Cell culture and treatment for VEGF assay}

Human normal keratinocytes (isolated from biopsy obtained from surgical operation) were plated in 96-well microplate at the density of 20,000 cells/well (culture medium KSFM). Plate was placed in an incubator maintained at $37^{\circ} \mathrm{C}$ and $5 \% \mathrm{CO}_{2}$ until cells were confluent at $80 \%$. Cells were treated at different concentrations in culture medium for 24 hours and then stimulated with IL-1 beta $(10 \mathrm{ng} / \mathrm{mL})$ for 24 hours. Each product concentration was evaluated in three wells stimulated or unstimulated. Each product concentration was evaluated in four wells. Cells were kept without treatment for control condition. At the end of the experiment, cell supernatants were frozen at $-20^{\circ} \mathrm{C}$ for subsequent analysis.

\section{VEGF quantification}

VEGF was measured as described in the VEGF enzymelinked immunosorbent assay kit (EHVEGF; Thermo Fisher Scientific). The spectrophotometric absorbance was measured using a scanning multiwell spectrophotometer (Safire). The wavelength to measure absorbance of the substrate was $450 \mathrm{~nm}$. A best fit curve was generated for the standards in order to extrapolate relative samples' values. The amount of VEGF was expressed in $\mathrm{pg} / \mathrm{mL}$. The percentages of inhibition induced by the treatment were calculated for each concentration tested in the presence or absence of the stimuli. Determination of protein was performed in each well using the bicinchoninic acid assay. VEGF quantification was expressed in $\mathrm{pg} / \mathrm{mL} / \mu \mathrm{g}$ protein. Values from three wells treated with the same condition were averaged. This average was compared with control average.

\section{In vitro angiogenesis assay}

Skin endothelial cells are a good model for angiogenesis study because when these cells are cultured on Matrigel ${ }^{\mathrm{TM}}$ (a three-dimensional support; BD Biosciences, San Jose, CA, USA), they tend to grow and associate themselves to create new vessels (tube like).

Skin endothelial cells were prepared by Kieda's laboratory according to their published protocol. ${ }^{10}$

Angiogenesis assay was performed on 96-well plates coated with $50 \mu \mathrm{L}$ of Matrigel ${ }^{\mathrm{TM}}$. After polymerization at $37^{\circ} \mathrm{C}$, skin endothelial cells (HSkMEC) were seeded at $1.5 \times 10^{4}$ cells per well. The plate was introduced into the incubation chamber of the video Zeiss Axiovert 200M fluorescence inverted microscope (Zeiss, Le Pecq, France) equipped with an Axiocam (high-resolution numeric camera) linked to a computer driving the acquisition software Axiovision (Zeiss). The direct real-time visualization of the formation of pseudovessels and network structures was monitored for 24 hours. 


\section{Study on human volunteers}

The efficacy of a serum containing RGC active vs placebo was done under dermatological control on 20 Caucasian women (mean age: $53 \pm 12$ years old) having subtype I rosacea, ie, erythematotelangiectatic rosacea.

International Nomenclature of Cosmetic Ingredients formula of the serum tested containing RGC active ingredient is aqua, glycerin, isostearyl isostearate, camellia oleifera seed oil, squalane, caprylic/capric triglyceride, glyceryl stearate citrate, butyrospermum parkii, polyglyceryl stearate citrate, behenyl alcohol, xanthan gum, hydrogenated lecithin, chlorphenesin, methylparaben, tocopherol, propylparaben, helianthus annuus seed oil, rosmarinyl glucoside, potassium hydroxide, gallyl glucoside, caffeyl glucoside, and citric acid.

Placebo serum used was made using the same formula without the following three components: rosmarinyl glucoside, gallyl glucoside, and caffeyl glucoside.

Volunteers were selected from a Caucasian population in this study, as rosacea occurs frequently in light-skinned, middle-aged individuals. The recommendations of the Declaration of Helsinki and the guidelines of the International Conference on Harmonization of Good Clinical Practice were observed as applicable to a nondrug study. All volunteers provided written informed consent. The volunteers were not subjected to any concomitant treatment that could potentially modify their skin condition. The volunteers used the serum and placebo on half their faces, every day, twice daily for 28 days. Biometrical analysis was done using a siascope tool. Siascope measures key chromophores within the skin, in particular hemoglobin in this study.

The various biometric measurements as well as pictures were taken during the first visit and after 28 days of twicedaily application. The measurements were taken in a controlled atmosphere room $\left(22^{\circ} \mathrm{C} \pm 2^{\circ} \mathrm{C}\right)$, after stabilization of the volunteers for at least 10 minutes. The various biometric measurements as well as pictures were taken from both cheekbone areas. The biometrological measurements were performed in the same way and on the same area before (D0) and after the treatment period (D28).

\section{Statistical analysis}

For all in vitro and in vivo studies, means and standard deviations were calculated. Then, the variation percentage compared to the initial value or to the control was calculated. In order to determine whether the evolution noticed is significant or not, a Student's $t$-test on paired samples was performed. The assumptions are the randomness and normal distribution of the samples. The threshold used were the following significant difference at $95 \%$ if $P<0.05$ and at $99 \%$ if $P<0.01$.

\section{Results \\ Cytotoxicity studies}

RGC tested up to $2.5 \%$ on all cells types was not cytotoxic. Each individual molecules (R, G, and $\mathrm{C}$ ) tested up to $1 \%$ were not cytotoxic (concentrations close to $90 \mathrm{mM}, 100 \mathrm{mM}$, and $130 \mathrm{mM}$, respectively).

\section{Cytokines release inhibition (IL-I beta and TNF alpha)}

Nonstimulated normal human keratinocytes were used in this experiment as normal controls, reflecting noninflammatory condition. IL-1 beta and PMA-stimulated keratinocytes mimicked the inflammatory condition.

Under nonstimulated conditions, keratinocytes spontaneously release IL-8 at a basal level in the culture media (Figure 1) and do not express TNF alpha or express TNF alpha at an undetectable level (Figure 2).

After keratinocytes treatment by IL-1 beta, a strong stimulation of IL-8 release was detected. Keratinocytes previously treated with $10 \mu \mathrm{M}$ dexamethasone and then stimulated by IL-1 beta showed a strong decrease in IL-8 release in the culture media $(-57 \%)$. These results validated the experimental model.

RGC tested from $1 \%$ to $2.5 \%$ inhibited IL-8 release upon cell stimulation markedly and significantly (Figure 1). At 1\% and $2.5 \%$, the inhibition percentages were, respectively, $43 \%$ and $67 \%(P<0.01)$ and demonstrated an inhibitory effect that was concentration dependent. We noticed that when RGC is applied at $2.5 \%$ on keratinocytes, the level of cytokine release in the medium is very close to those measured on unstimulated keratinocytes.

After keratinocytes treatment by PMA, a stimulation of TNF alpha release was detected. Thus, when keratinocytes were previously treated with dexamethasone at $10 \mu \mathrm{M}$ and then stimulated by PMA, a strong decrease in TNF alpha release in the culture media was recorded $(-59 \%)$. These results validate the experiments. RGC at $1 \%, 1.5 \%$, and $2.5 \%$ inhibits TNF alpha release, respectively, by $30 \%, 39 \%$, and $37 \%$ ( $P<0.01$, Figure 2$)$. The inhibitory effect observed with RGC was not concentration dependent.

\section{Histamine release inhibition}

Under nonstimulated condition, mast cells released histamine in the culture media spontaneously at a low level (Figure 3). After their contact with substance P, mast cells 


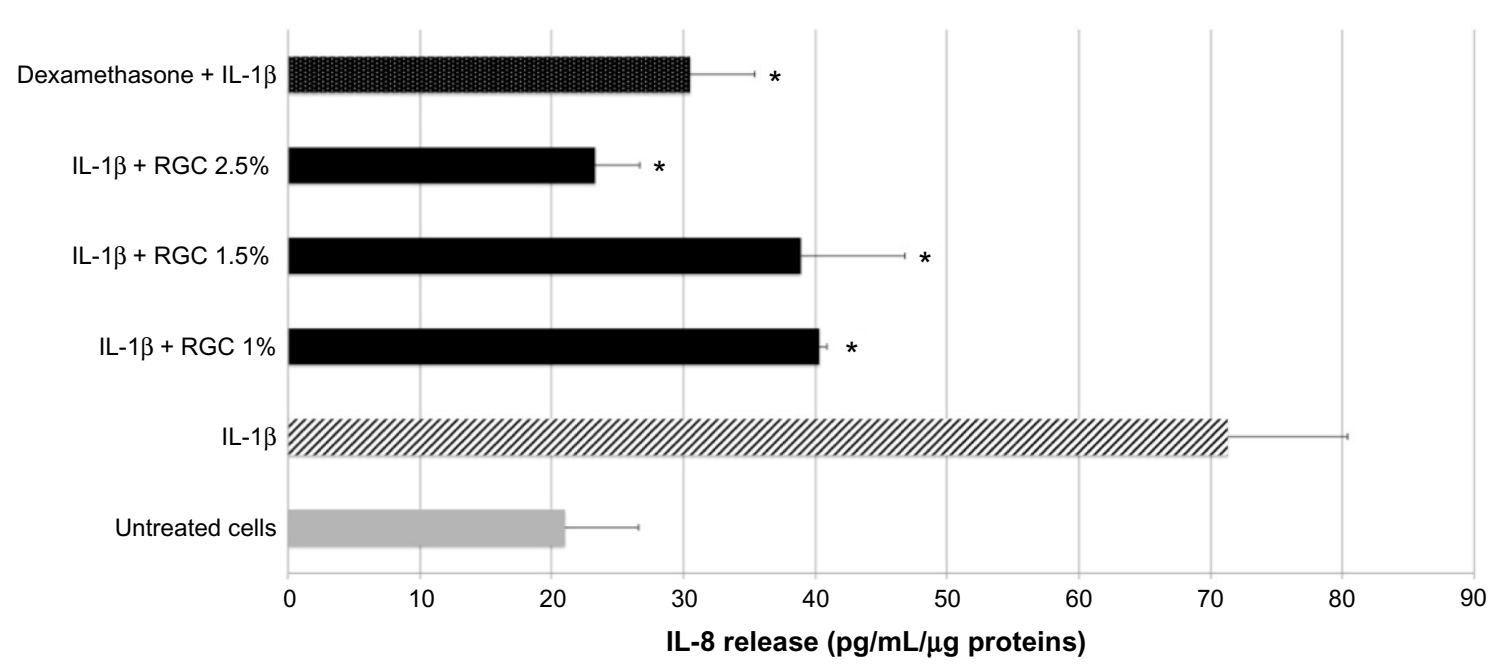

Figure I Effect of RGC active on interleukin-8 (IL-8) release from normal human keratinocytes.

Notes: Human keratinocytes were pretreated with RGC at I\%, I.5\%, and $2.5 \%$ for 24 hours and then incubated with interleukin-I beta (IL-I beta) at I ng/mL during 24 hours. IL-8 release was assessed using Quantikine assay. Values are mean \pm standard deviation $(n=3)$. Significance was determined by Student's $t$-test. *Corresponding to the statistical significance calculated with $P<0.01$.

Abbreviation: RGC, rosmarinic acid glucoside (R), gallic acid glucoside (G), and caffeic acid glucoside (C).

released histamine in abundant quantity in comparison to untreated condition $(+235 \%)$. Mast cells pretreated with calcium at $10 \mathrm{mM}$ and then with substance $\mathrm{P}$ showed a strong decrease in histamine release in the culture media $(-68 \%)$. Calcium pretreatment fully annihilates substance $\mathrm{P}$ action. These results validated the experimental model.

Mast cells pretreated with RGC at $1 \%, 1.5 \%$, and $2.5 \%$ before substance $\mathrm{P}$ addition induced a significant inhibition of histamine release (from $15 \%$ to $21 \%$ of inhibition, Figure 3), without reaching the basal release level seen with untreated cells.

\section{Angiogenesis inhibition studies}

Once treated with IL-1, VEGF release in culture media was increased $(+12 \%)$. Thus, when keratinocytes were previously treated with dexamethasone at $10 \mu \mathrm{M}$ and then stimulated by IL-1 beta, a significant and strong decrease in VEGF release in the culture media was recorded $(-56 \%, P<0.01)$. These results validated the experimental model. RGC treatments at $1.5 \%$ and $2.5 \%$ inhibited VEGF release induced by IL-1 beta, respectively, by $33 \%$ and $37 \%$ in comparison to stimulated condition $(P<0.01$, Figure 4$)$. VEGF level assessed in culture media after RGC treatment was less than the basal level (untreated keratinocytes).

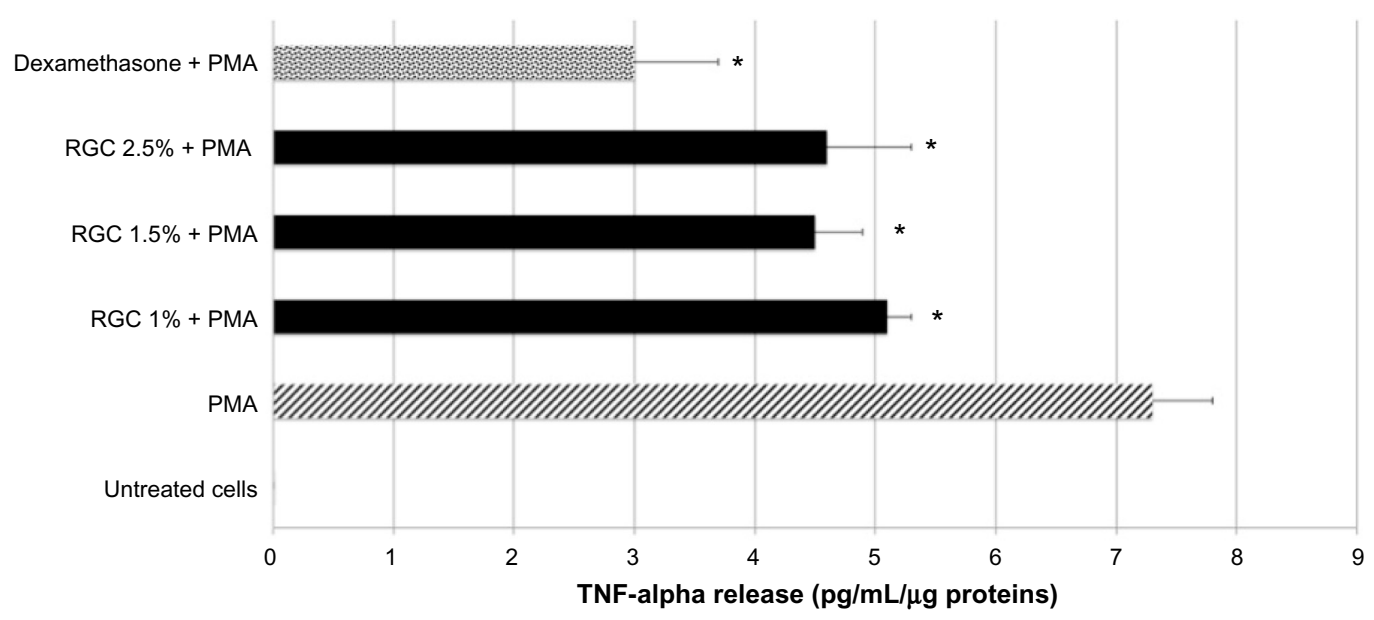

Figure 2 Effect of RGC on tumor necrosis factor alpha (TNF alpha) release from normal human keratinocytes under stimulated condition.

Notes: Human keratinocytes were pretreated with RGC at $1 \%$, I.5\%, and $2.5 \%$ for 24 hours and then incubated with phorbol myristate acetate (PMA) at 500 ng/mL during 24 hours. TNF alpha release was assessed using Quantikine assay. Values are mean \pm standard deviation $(n=3)$. Significance was determined by Student's $t$-test. *Corresponding to the statistical significance calculated with $P<0.01$.

Abbreviation: RGC, rosmarinic acid glucoside (R), gallic acid glucoside $(\mathrm{G})$, and caffeic acid glucoside (C). 


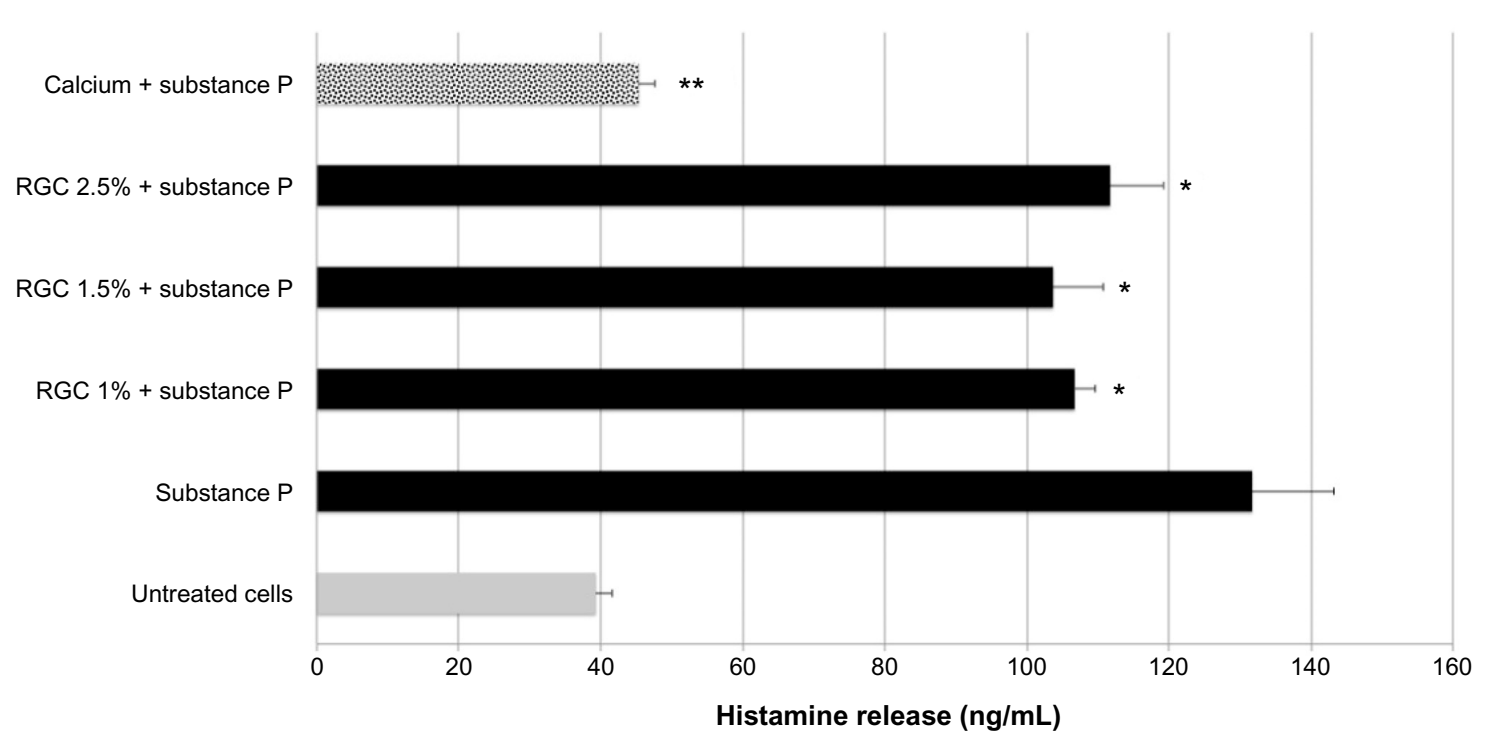

Figure 3 Effect of RGC on histamine release from mast cells under normal and stimulated conditions.

Notes: Mast cells were pretreated with RGC at I\%, I.5\%, and $2.5 \%$ for 30 minutes and then incubated with substance P at $50 \mu \mathrm{M}$ for 15 minutes. Histamine release was assessed using ELISA assay. Values are mean \pm standard deviation $(n=3)$. Significance was determined by Student's $t$-test. $*$ Corresponding to the statistical significance calculated with $P<0.05$ and **with $P<0.01$. Stimulated condition was done using substance $P$.

Abbreviations: RGC, rosmarinic acid glucoside (R), gallic acid glucoside (G), and caffeic acid glucoside (C); ELISA, enzyme-linked immunosorbent assay.

Angiogenesis is characterized by the formation of new vessels under VEGF stimulation. RGC tested at $2.5 \%$ has shown the capability to inhibit the formation of new vessel

\section{(Figure 5).}

RGC clearly showed an inhibitory effect on the formation of neovessels. This effect is not due to a potential cytotoxic effect of RGC, as our studies have shown its noncytotoxicity.

\section{In vivo study}

After 28 days of treatment, the half face treated with the placebo showed either no evolution of the rosacea or a negative evolution of the symptoms. On the half face treated with $2 \%$ RGC serum, a clear reduction in rosacea skin conditions was seen (Figure 6). The visible symptoms were reduced up to $-12 \%$ for $30 \%$ of the volunteers, and no side effects were reported. The improvement in rosacea symptoms (decreasing them) after

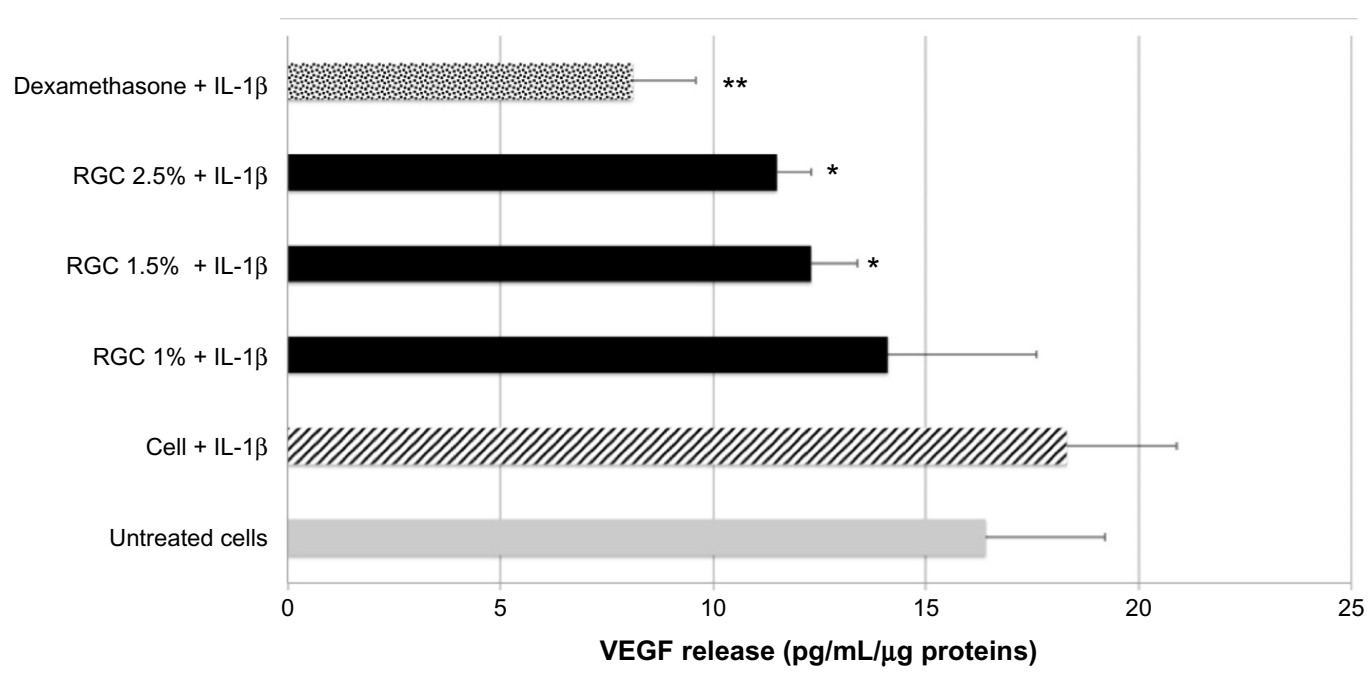

Figure 4 Effect of RGC on VEGF release from keratinocytes.

Notes: Human keratinocytes were pretreated with RGC at I\%, I.5\%, and 2.5\% for 24 hours and then incubated with IL-I beta at I ng/mL during 24 hours. VEGF release was assessed using ELISA assay. Values are mean \pm standard deviation $(n=3)$. Significance was determined by Student's $t$-test. *Corresponding to the statistical significance calculated with $P<0.05$ and **with $P<0.01$.

Abbreviations: RGC, rosmarinic acid glucoside (R), gallic acid glucoside (G), and caffeic acid glucoside (C); VEGF, vascular endothelial growth factor; IL, interleukin; ELISA, enzyme-linked immunosorbent assay. 


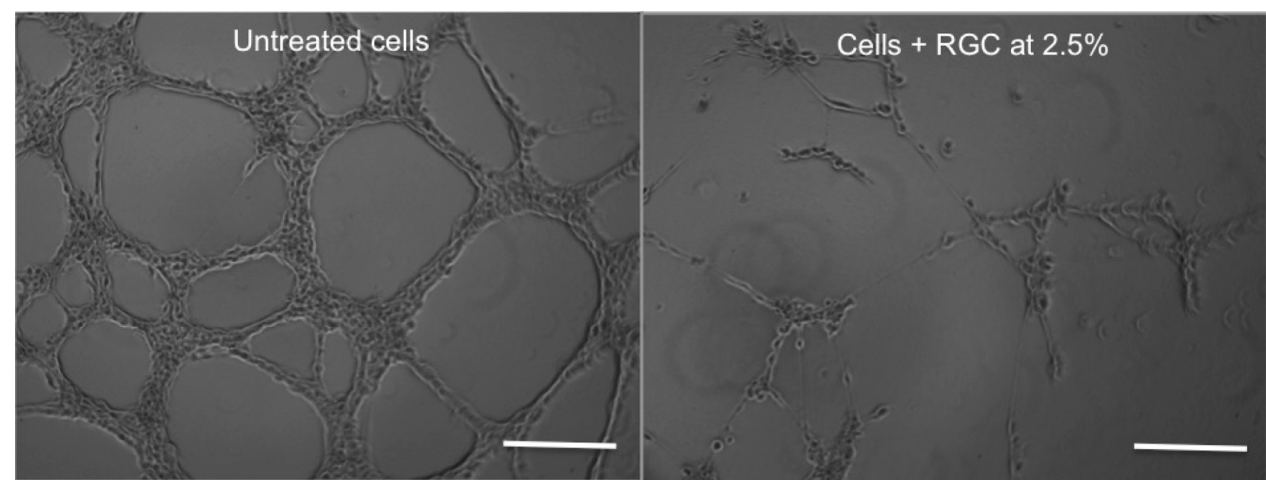

Figure 5 Effect of RGC on the angiogenesis process in vitro.

Notes: Skin endothelial cells (HSkMEC) were treated or not with RGC at $2.5 \%$ and seeded on Matrigel ${ }^{\mathrm{TM}}$. Scale bars represent I00 $\mu \mathrm{m}$.

Abbreviation: RGC, rosmarinic acid glucoside (R), gallic acid glucoside (G), and caffeic acid glucoside (C).

RGC treatment was observed for $50 \%$ of the volunteers. In the other half of the population having RGC serum treatment, rosacea skin condition was not worsened.

\section{Discussion}

Current management of all types of rosacea disorders includes patient education (avoidance of triggers, such as sun exposure and spicy foods), use of proper skin care, and depending on the type of rosacea, pharmacological drugs having either topical or systemic action. ${ }^{11}$ Medical devices (laser and light therapies) and surgery are also proposed by physicians. $^{12-15}$

Moreover, due to chronicity and multifactorial origin of this disease, rosacea management needs time and combined drugs and/or medical devices approach. Surgery is a recommended solution for phymatous rosacea.

Traditional topical therapies using drugs are related to azelaic acid, metronidazole, Ivermectin ${ }^{\circledR}$, whereas systemic drug therapies concern tetracyclines, isotretinoin, and also Ivermec$\operatorname{tin}^{\circledR}$. These drugs are only used for papulopustular rosacea and ocular rosacea. ${ }^{16,17}$ Azelaic acid and metronidazole act on the inflammation by inhibiting neutrophils reactive oxygen species production. ${ }^{18}$ Isotretinoin gets antiseborrheic activity and antiinflammatory properties. ${ }^{19}$ Ivermectin $^{\circledR}$, an antiparasitic drug, was proposed for papulopustular rosacea recently because recent scientific papers have demonstrated that Demodex folliculorum mites are involved in this type of rosacea. ${ }^{20}$

Recently in 2014, Brimonidine ${ }^{\circledR}$, a FDA-approved drug, was proposed for the management of erythematotelangiectatic rosacea as a topical therapy. ${ }^{21}$ Brimonidine ${ }^{\circledR}$ acts by reversing skin vasodilation. Alternative methods to drugs for papulopustular rosacea or erythematotelangiectatic rosacea are based either on the use of dermocosmetic or on the medical devices solutions (laser and intense pulsed light therapies). Dermocosmetic solutions imply the use of lotion composed of botanical extract ${ }^{22}$ or onabotulinumtoxin A. ${ }^{23}$ Laser and intense pulsed light therapies are considered as emerging therapies and were reported to be beneficial for the treatment of erythema and telangiectasia, as well as for some rosacea symptoms, such as itching, burning, pain, stinging, and swelling.

Until now, none of these solutions in the market used alone is fully curative for rosacea disorders, regardless of the rosacea type concerned, and so a new potent dermocosmetic ingredient could be useful for this already available armamentarium. Based on this observation and on the current knowledge of rosacea physiopathology, we decided to develop a dermocosmetic ingredient that can relieve symptoms of erythematotelangiectatic rosacea in order to avoid the worsening of the situation.

The choice of molecules to form the active ingredient was based on the literature analysis, showing that caffeic acid, rosmarinic acid, and gallic acid get interesting biological properties that could be useful to slow down the pathological process of rosacea. Anti-angiogenesis function of caffeic acid was demonstrated on retinal tissues. ${ }^{24}$ Rosmarinic acid is a molecule having anti-inflammation ${ }^{25}$ and anti-degranulation ${ }^{26}$ action, two interesting properties of rosacea. Tsai et al have shown that rosmarinic acid suppresses IL-8 release. ${ }^{27}$ Thus, gallic acid like rosmarinic acid also has an anti-inflammation and an anti-degranulation action. ${ }^{28} \mathrm{Kang}$ et al also found the inhibitory effect of gallic acid on IL-8 release in oral epithelial cells. ${ }^{29}$

Moreover, there are no published data showing that these components were already used in rosacea pathology, but due to their known instability and difficulty to formulate, we decided to add a glucosyl group to these three molecules by enzymatic reaction to create new stable, water soluble, and 


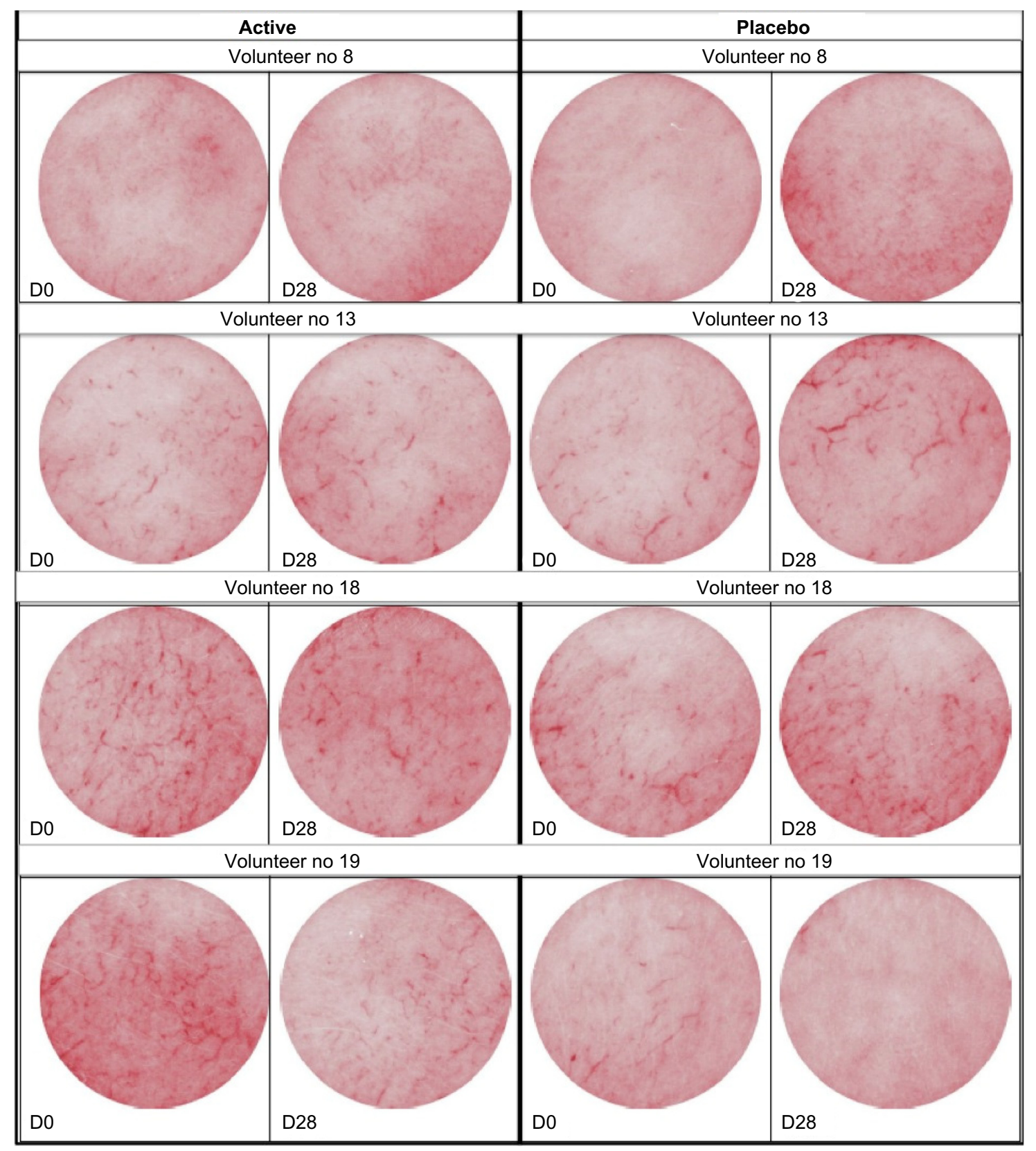

Figure 6 Pictures showing the skin condition of four volunteers before and after 2\% RGC serum (active) or placebo treatment during 28 days (Siagraphs). Abbreviations: RGC, rosmarinic acid glucoside (R), gallic acid glucoside (G), and caffeic acid glucoside (C); D, day; no, number.

easy to formulate molecules. Previously published data from our laboratory ${ }^{30}$ demonstrated that glucosylation of gallic acid does not modify its biological properties. We found using cellular studies that RGC has anti-inflammatory, anti-angiogenic, and anti-degranulation properties. RGC decreases significantly IL-1, TNF alpha, and VEGF release from keratinocytes. In vivo, cytokines, such as IL-1 beta or TNF alpha, modulate the activity of VEGF, a major angiogenic factor. In our experiments, we confirmed like other authors ${ }^{31-33}$ that normal human keratinocytes express a basal level of VEGF. We have also shown that VEGF is overexpressed under inflammatory condition. Interestingly, RGC was able to decrease VEGF expression level under this inflammatory condition due to its action on TNF alpha production. VEGF release is known to be mediated by TNF alpha.

Moreover, RGC through its mast cells anti-degranulation properties could also control the angiogenesis seen in rosacea. RGC inhibits the formation of neovessels, suggesting that this product could hinder the formation of new blood vessels in irritated skin and could improve vascular disorders linked to cutaneous redness or blotches such those seen in rosacea. It is known that under pathological conditions, VEGF released by 
keratinocytes stimulates the formation of neovessels by endothelial cells. The positive effects observed by topical application of RGC in vivo could be explain in part by this mechanism.

Internal unpublished data on caffeic acid glucoside have shown its capability to decrease PGE2 release. This property is interesting as PGE2 release is involved in vasodilation, edema, and erythema. We could assume, according to these in vitro findings, that our clinical observations showing that RGC helps to limit deteriorating skin condition is through the synergic action of glucosylated form and unglucosylated moieties of rosmarinic acid, gallic acid, and caffeic acid.

\section{Conclusion}

Although rosacea is a skin condition disorder difficult to heal, these studies have shown that RGC active could be a dermocosmetic help, especially for erythematotelangiectatic rosacea armamentarium. Indeed, $2 \%$ of RGC topically applied on face for 28 days improved erythematotelangiectatic rosacea symptoms either by decreasing them (in half of the volunteers, vessels are less visible) or by limiting their development (any neovessels seen). RGC acts by decreasing inflammation (inhibition of IL-8 and TNF release), decreasing degranulation of mast cells (inhibition of histamine release), and limiting the angiogenesis process (inhibition of VEGF production and neovessel formation).

\section{Acknowledgments}

The authors would like to thank Professor Claudine Kieda and Dr Catherine Grillon from CBM-CNRS (Orleans, France) for the studies done on endothelial cells. They also thank Dr Francine Joly (Sephra pharma, Puteaux, France) for cytokines and mast cells studies, Dr Elian Lati (Laboratoire Bio-EC, Longjumeau, France) for clinical study management, and Mrs Parand Salmassinia for the English language review.

\section{Disclosure}

The authors report no conflicts of interest in this work.

\section{References}

1. Korting HC, Schollmann C. Current topical and systemic approaches to treatment of rosacea. $J$ Eur Acad Dermatol Venereol. 2009;23(8): 876-882.

2. Fimmel S, Abdel-Naser MB, Kutzner H, Kligman AM, Zouboulis CC. New aspects of the pathogenesis of rosacea. Drug Discov Today Dis Mech. 2008;5(1):e103-e111.

3. Norrby K. Mast cells and angiogenesis. APMIS. 2002;110(5):355-371.

4. Smith JR, Lanier VB, Braziel RM, Falkenhagen KM, White C, Rosenbaum JT. Expression of vascular endothelial growth factor and its receptors in rosacea. Br J Ophthalmol. 2007;91(2):226-229.

5. Auriol Daniel NR, Robe Patrick, Lefevre Fabrice. Phenolic compounds with cosmetic and therapeutic applications. Patent EP 2027279B1. 2006 June 14.
6. Stoddart MJ. Cell viability assays: introduction. Methods Mol Biol. 2011;740:1-6.

7. Loo DT, Rillema JR. Measurement of cell death. Methods Cell Biol. 1998;57:251-264.

8. Berridge MV, Herst PM, Tan AS. Tetrazolium dyes as tools in cell biology: new insights into their cellular reduction. Biotechnol Annu Rev. 2005;11:127-152.

9. Rampersad SN. Multiple applications of Alamar Blue as an indicator of metabolic function and cellular health in cell viability bioassays. Sensors (Basel). 2012;12(9):12347-12360.

10. Kieda C, Paprocka M, Krawczenko A, et al. New human microvascular endothelial cell lines with specific adhesion molecules phenotypes. Endothelium. 2002;9(4):247-261.

11. Weinkle AP, Doktor V, Emer J. Update on the management of rosacea. Clin Cosmet Investig Dermatol. 2015;8:159-177.

12. Del Rosso JQ. Management of cutaneous rosacea: emphasis on new medical therapies. Expert Opin Pharmacother. 2014;15(14):2029-2038.

13. Moustafa FA, Sandoval LF, Feldman SR. Rosacea: new and emerging treatments. Drugs. 2014;74(13):1457-1465.

14. Bassichis BA, Swamy R, Dayan SH. Use of the KTP laser in the treatment of rosacea and solar lentigines. Facial Plast Surg. 2004;20(1): $77-83$.

15. Taub AF. Treatment of rosacea with intense pulsed light. $J$ Drugs Dermatol. 2003;2(3):254-259.

16. Elewski BE, Fleischer AB Jr, Pariser DM. A comparison of $15 \%$ azelaic acid gel and $0.75 \%$ metronidazole gel in the topical treatment of papulopustular rosacea: results of a randomized trial. Arch Dermatol. 2003;139(11):1444-1450.

17. Thielitz A, Gollnick H. [Isotretinoin. How should it be used?]. Hautarzt. 2013;64(4):263-268.

18. Wolf JE Jr, Kerrouche N, Arsonnaud S. Efficacy and safety of once-daily metronidazole $1 \%$ gel compared with twice-daily azelaic acid $15 \%$ gel in the treatment of rosacea. Cutis. 2006;77(4 Suppl):3-11.

19. Park H, Del Rosso JQ. Use of oral isotretinoin in the management of rosacea. J Clin Aesthet Dermatol. 2011;4(9):54-61.

20. Allen KJ, Davis CL, Billings SD, Mousdicas N. Recalcitrant papulopustular rosacea in an immunocompetent patient responding to combination therapy with oral ivermectin and topical permethrin. Cutis. 2007; 80(2):149-151.

21. Brimonidine for erythema caused by rosacea. Drug Ther Bull. 2014; 52(12):138-140.

22. Emer J, Waldorf H, Berson D. Botanicals and anti-inflammatories: natural ingredients for rosacea. Semin Cutan Med Surg. 2011;30(3):148-155.

23. Dayan SH, Pritzker RN, Arkins JP. A new treatment regimen for rosacea: onabotulinumtoxinA. J Drugs Dermatol. 2012;11(12):e76-e79.

24. Kim JH, Lee BJ, Kim JH, Yu YS, Kim KW. Anti-angiogenic effect of caffeic acid on retinal neovascularization. Vascul Pharmacol. 2009; 51(4):262-267.

25. Zdarilova A, Svobodova A, Simanek V, Ulrichova J. Prunella vulgaris extract and rosmarinic acid suppress lipopolysaccharide-induced alteration in human gingival fibroblasts. Toxicol In Vitro. 2009;23(3):386-392.

26. Oh HA, Park CS, Ahn HJ, Park YS, Kim HM. Effect of Perilla frutescens var. acuta Kudo and rosmarinic acid on allergic inflammatory reactions. Exp Biol Med. 2011;236(1):99-106.

27. Tsai TH, Chuang LT, Lien TJ, Liing YR, Chen WY, Tsai PJ. Rosmarinus officinalis extract suppresses Propionibacterium acnesinduced inflammatory responses. J Med Food. 2013;16(4):324-333.

28. Kim SH, Jun CD, Suk K, et al. Gallic acid inhibits histamine release and pro-inflammatory cytokine production in mast cells. Toxicol Sci. 2006;91(1):123-131.

29. Kang MS, Jang HS, Oh JS, et al. Effects of methyl gallate and gallic acid on the production of inflammatory mediators interleukin- 6 and interleukin- 8 by oral epithelial cells stimulated with Fusobacterium nucleatum. J Microbiol. 2009;47(6):760-767.

30. Chajra H, Auriol D, Schweikert K, Lefevre F. Targeting inflammatory pathways to reduce dark circles and puffiness. SOFW J. 2014;140(4): $16-31$. 
31. Viac J, Palacio S, Schmitt D, Claudy A. Expression of vascular endothelial growth factor in normal epidermis, epithelial tumors and cultured keratinocytes. Arch Dermatol Res. 1997;289(3):158-163.

32. Weninger W, Uthman A, Pammer J, et al. Vascular endothelial growth factor production in normal epidermis and in benign and malignant epithelial skin tumors. Lab Invest. 1996;75(5):647-657.
33. Trompezinski S, Berthier-Vergnes O, Denis A, Schmitt D, Viac J. Comparative expression of vascular endothelial growth factor family members, VEGF-B, -C and -D, by normal human keratinocytes and fibroblasts. Exp Dermatol. 2004;13(2):98-105.

\section{Publish your work in this journal}

Clinical, Cosmetic and Investigational Dermatology is an international, peer-reviewed, open access, online journal that focuses on the latest clinical and experimental research in all aspects of skin disease and cosmetic interventions. All areas of dermatology will be covered; contributions will be welcomed from all clinicians and basic science researchers globally. This journal is indexed on CAS

The manuscript management system is completely online and includes a very quick and fair peer-review system, which is all easy to use. Visit http://www.dovepress.com/testimonials.php to read real quotes from published authors.

Submit your manuscript here: http://www.dovepress.com/clinical-cosmetic-and-investigational-dermatology-journal 